\title{
A comparison of computerized tomography findings of COVID-19 infection by gender and age groups
}

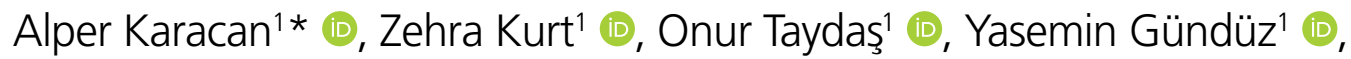

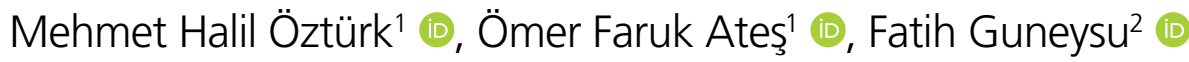

\section{SUMMARY}

OBJECTIVE: To compare the computed tomography (CT) imaging findings of coronavirus disease 2019 (COVID-19) by gender and age groups. METHODS: The patients with COVID-19 ( $n=1,024)$ were divided into nine age groups (0-9 years, 10-19 years, 20-29 years, 30-39 years, 40-49 years, 50-59 years, 60-69 years, 70-79 years, and 80 years and above). The CT findings were retrospectively analyzed according to the age groups and gender.

RESULTS: Under 20 years of age, except for the ground-glass opacity and consolidation, no other finding was observed. Airway changes and crazy-paving pattern were more common over 80 years. While the tree-in-bud pattern was more common in the 20-29 age group than in other age groups, the halo sign was mostly seen at the age of 30-39 years. Unlike other groups, the thin reticular pattern was more common in patients aged 60-79 years. When the findings were compared by gender, the rates of centrilobular nodules ( $p=0.006)$, airway changes ( $p=0.004)$, and tree-in-bud pattern ( $p=0.050)$ were significantly higher in males than in females.

CONCLUSION: The chest CT findings of COVID-19 show significant changes according to age and gender. The findings that are more common in elderly and male patients should be carefully evaluated in terms of the prognosis of the disease.

KEYWORDS: COVID-19. Computerized tomography. Age. Gender.

\section{INTRODUCTION}

The novel coronavirus disease 2019 (COVID-19) is an infectious disease, which was first identified in December 2019 and declared a pandemic in March 2020, spreading rapidly to the whole world and causing the death of $1,280,000$ people as of November 11, 2020 ${ }^{1}$. COVID-19 is a viral disease that spreads rapidly from person to person and can cause serious health problems, such as pneumonia, necrotizing encephalopathy, systemic and pulmonary thromboembolism, acute respiratory distress syndrome, respiratory failure, and sepsis ${ }^{1-3}$. Approximately, 15-20\% of patients have the serious form of the disease, and the mortality rate is around $2-3 \%{ }^{4}$.

Recent studies have highlighted the importance of a chest CT examination in COVID-19 patients with false negative RT-PCR results and reported CT sensitivity as $98 \%$,5.6. However, chest CT images may show different imaging features in COVID-19 patients depending on the stage and severity of the disease ${ }^{7}$.

The typical CT features of COVID-19 pneumonia are bilateral and multifocal ground-glass opacities (GGO). Classically, lesions are predominant in the peripheral, posterior, and basal parts of the lungs ${ }^{8}$. Despite the large number of

\footnotetext{
'Sakarya University Faculty of Medicine, Department of Radiology - Sakarya, Turkey.

${ }^{2}$ Sakarya University Faculty of Medicine, Department of Emergency - Sakarya, Turkey.

*Corresponding author: alperkaracan@sakarya.edu.tr

Conflicts of interest: the authors declare there are no conflicts of interest. Funding: none.

Received on March 03, 2021. Accepted on March 14, 2021.
} 
studies on the CT findings of COVID-19, there are only limited studies evaluating the distribution of findings by gender and age. Most of the available studies present the comparison of pediatric and adult patients without age grouping.

The aim of this study was to evaluate the distribution of the CT findings of the COVID-19 disease by age and gender.

\section{METHODS}

This retrospective study was carried out with 1,024 patients who were admitted in the Sakarya University Education and Research Hospital between January 1 and April 1, 2020 with the suspicion of COVID-19. The study protocol was approved by the local ethics committee of the Faculty of Medicine of the University (IRB No. 71522473/050.01.04/221). The RT-PCR assay of the nasopharyngeal or oropharyngeal swab samples and chest CT imaging data of 1,024 patients were recorded within the scope of the study. The inclusion criteria were having a positive PCR-RT test and complete CT findings. The exclusion criteria were having a chronic lung disease or a history of any lung pathology. The sample was divided into nine groups based on age decades.

\section{Computed tomography protocol}

For the investigation of COVID-19 pneumonia, all the patients underwent nonenhanced chest $\mathrm{CT}$ examinations in the supine position during end-inspiration. The CT scans of the patients were obtained with a 64-section multidetector CT scanner (Aquilion 64, Toshiba, Japan) using the following protocols: tube voltage $120 \mathrm{kV}$, automatic tube current $120-380 \mathrm{~mA}$, thickness $5 \mathrm{~mm}$, slice interval $5 \mathrm{~mm}$, rotation speed $0.5 \mathrm{~s}$, and helical pitch 1.0875:1 or 1.375:1 for the adults, and tube voltage $100 \mathrm{kV}$, automatic tube current $30-100 \mathrm{~mA}$, thickness 3-5 mm, slice interval $1 \mathrm{~mm}$, rotation speed $0.6 \mathrm{~s}$, and helical pitch 0.969:1 for the pediatric patients. Lung window images at $0.625-1 \mathrm{~mm}$ thickness were reconstructed using the iterative technique. The informed consent for the CT examination was obtained from all patients or from their parents. The chest CT features were reviewed by two radiologists (AK and YG with 10 and 15 years of experience in thoracic imaging, respectively) based on consensus. Any controversy between the two radiologists evaluating thorax CT findings was resolved by consulting a third experienced radiologist (MHO with more than 20 years of experience in thoracic imaging). The imaging findings were evaluated comparatively by gender and age groups.

\section{Statistical analysis}

Statistical analysis was performed with SPSS v23.0 (IBM, New York, NY, USA), and $\mathrm{p}<0.05$ indicated a statistically significant difference. Quantitative variables were expressed as mean or median values and standard deviation. Categorical variables were compared with the Pearson's chi-square test.

\section{RESULTS}

A total of 1,024 patients, 546 men (53.32\%) and 478 women (46.68\%), were included in the study. The average age was $46.29 \pm 17.99$ years. The distribution of the CT findings is shown in Figure 1. GGO was present in 715 patients $(69.82 \%)$, consolidation in 350 (34.18\%), intra-interlobular septal thickening in $152(14.84 \%)$, crazy-paving pattern in $96(9.38 \%)$, thin reticular pattern in $86(8.40 \%)$, airway changes in $72(7.03 \%)$, air bronchogram in 62 (6.05\%), fibrosis in 48 (4.69\%), lymphadenopathy in 35 (3.42\%), intraparenchymal vascular enlargement in $28(2.73 \%)$, pleural effusion in 25 (2.44\%), air bubbles in $21(2.05 \%)$, tree-in-bud pattern in $20(1.95 \%)$, halo sign in $20(1.95 \%)$, reverse halo sign in $17(1.66 \%)$, centrilobular nodule in $16(1.56 \%)$, and pericardial effusion in $11(1.07 \%)$.

The results of the comparison of the CT findings by gender are given in Table 1. In the males, the rates of centrilobular nodules $(2.56 \%)$, airway changes $(9.16 \%)$, and tree-inbud pattern $(2.75 \%)$ were significantly higher compared to the females $(0.42,4.60$, and $1.05 \%$, respectively) $(\mathrm{p}=0.006,0.004$, and 0.050 , respectively). The statistically significant findings according to the gender comparison are shown in Figure 2.

When the CT findings were compared between the age groups, there were significant differences in terms of the rates of GGO, consolidation, intra-interlobular septal thickening, thin reticular pattern, fibrosis, extrapulmonary involvement (pleural effusion), extrapulmonary involvement (lymphadenopathy), air bubble, air bronchogram, airway changes, pericardial effusion, and crazy-paving pattern. The results of the comparison of the CT findings by age are given in Table 2 .

Under 20 years of age, except for the GGO and consolidation, no other finding was observed.

The airway changes and crazy-paving pattern were more common over 80 years.

While the tree-in-bud pattern was most frequently seen in the 20-29 years age group, the halo sign was more common in the patients aged 30-39 years compared to the other age groups. Unlike other ages, the thin reticular pattern was more common in the 60-79 years age group. The higher frequencies of all these findings in different age groups were statistically significant $(\mathrm{p}<0.001)$.

Some of the CT findings are shown in Figure 3.

Furthermore, the chi-square trend analysis revealed that the significant relationship of the CT findings with age had an increasing trend as the age progressed. 


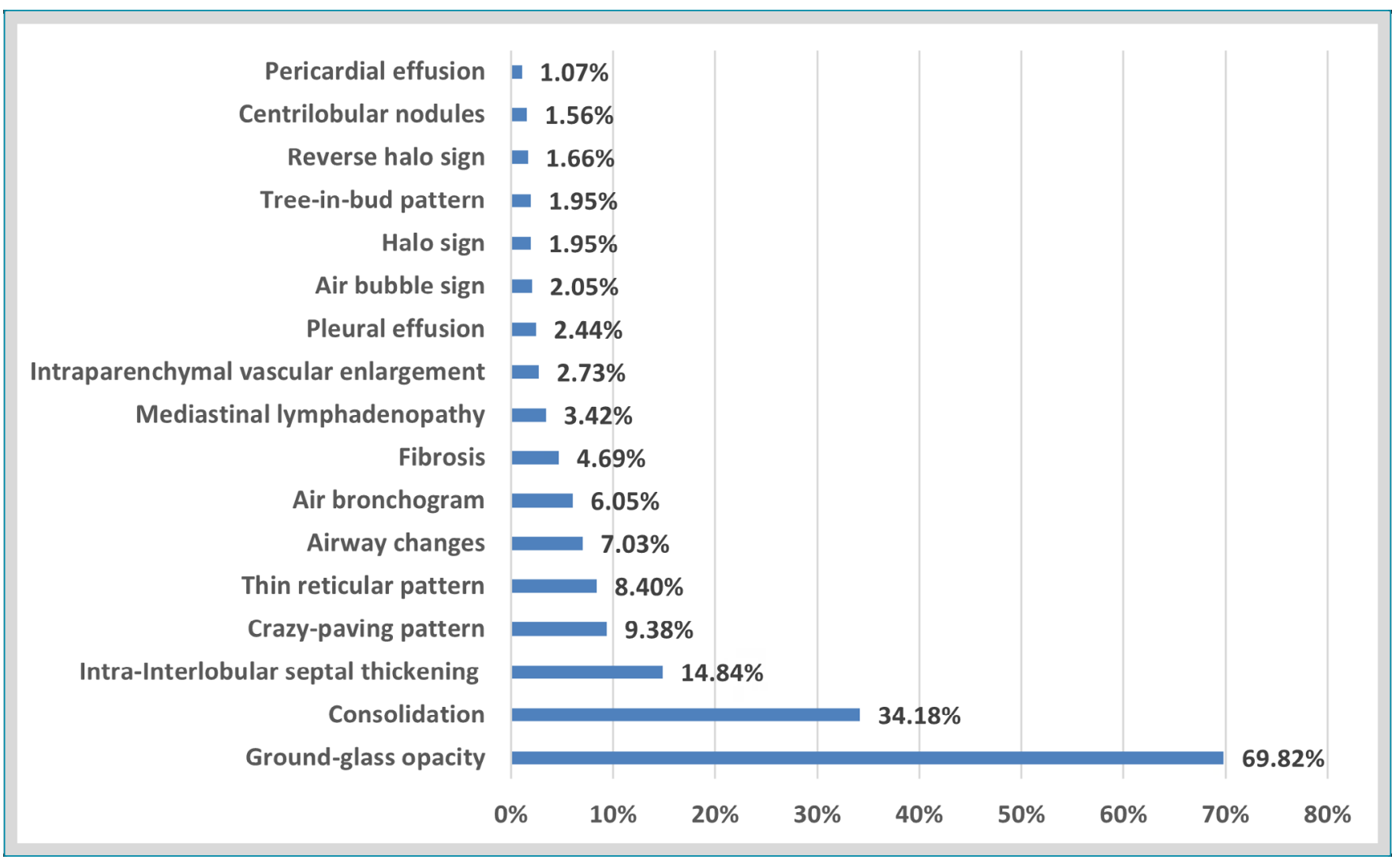

Figure 1. Distribution of computed tomographic findings of COVID-19.

Table 1. Distribution of parenchymal abnormalities according to gender.

\begin{tabular}{l|c|c|c|c|c}
\multirow{2}{*}{} & \multicolumn{2}{c|}{ Male } & \multicolumn{2}{c|}{ Female } & \multirow{2}{*}{ p-value } \\
\cline { 2 - 5 } & $\mathrm{n}$ & $\%$ & $\mathrm{n}$ & $\%$ & \multirow{2}{*}{ Ground-glass opacity } \\
\cline { 2 - 5 } & 384 & $(70.3)$ & 331 & $(69.25)$ & 0.706 \\
\hline Consolidation & 185 & $(33.88)$ & 165 & $(34.52)$ & 0.830 \\
\hline Intra-interlobular septal thickening & 89 & $(16.30)$ & 63 & $(13.18)$ & 0.161 \\
\hline Thin reticular pattern & 52 & $(9.52)$ & 34 & $(7.11)$ & 0.165 \\
\hline Centrilobular nodules & 14 & $(2.56)$ & 2 & $(0.42)$ & $0.006^{*}$ \\
\hline Intraparenchymal vascular enlargement & 20 & $(3.66)$ & 8 & $(1.67)$ & 0.051 \\
\hline Halo sign & 8 & $(1.47)$ & 12 & $(2.51)$ & 0.228 \\
\hline Reverse halo sign & 11 & $(2.01)$ & 6 & $(1.26)$ & 0.343 \\
\hline Fibrosis & 30 & $(5.49)$ & 18 & $(3.77)$ & 0.192 \\
\hline Pleural effusion & 13 & $(2.38)$ & 12 & $(2.51)$ & 0.893 \\
\hline Mediastinal lymphadenopathy & 23 & $(4.21)$ & 12 & $(2.51)$ & 0.135 \\
\hline Air bubble sign & 13 & $(2.38)$ & 8 & $(1.67)$ & 0.426 \\
\hline Air bronchogram & 39 & $(7.14)$ & 23 & $(4.81)$ & 0.119 \\
\hline Airway changes & 50 & $(9.16)$ & 22 & $(4.60)$ & $0.004^{*}$ \\
\hline Pericardial effusion & 3 & $(0.55)$ & 8 & $(1.67)$ & 0.082 \\
\hline Tree-in-bud pattern & 15 & $(2.75)$ & 5 & $(1.05)$ & $0.050^{*}$ \\
\hline Crazy-paving pattern & 54 & $(9.89)$ & 42 & $(8.79)$ & 0.546 \\
\hline
\end{tabular}

${ }^{*} p<0.05$ is statistically significant. 


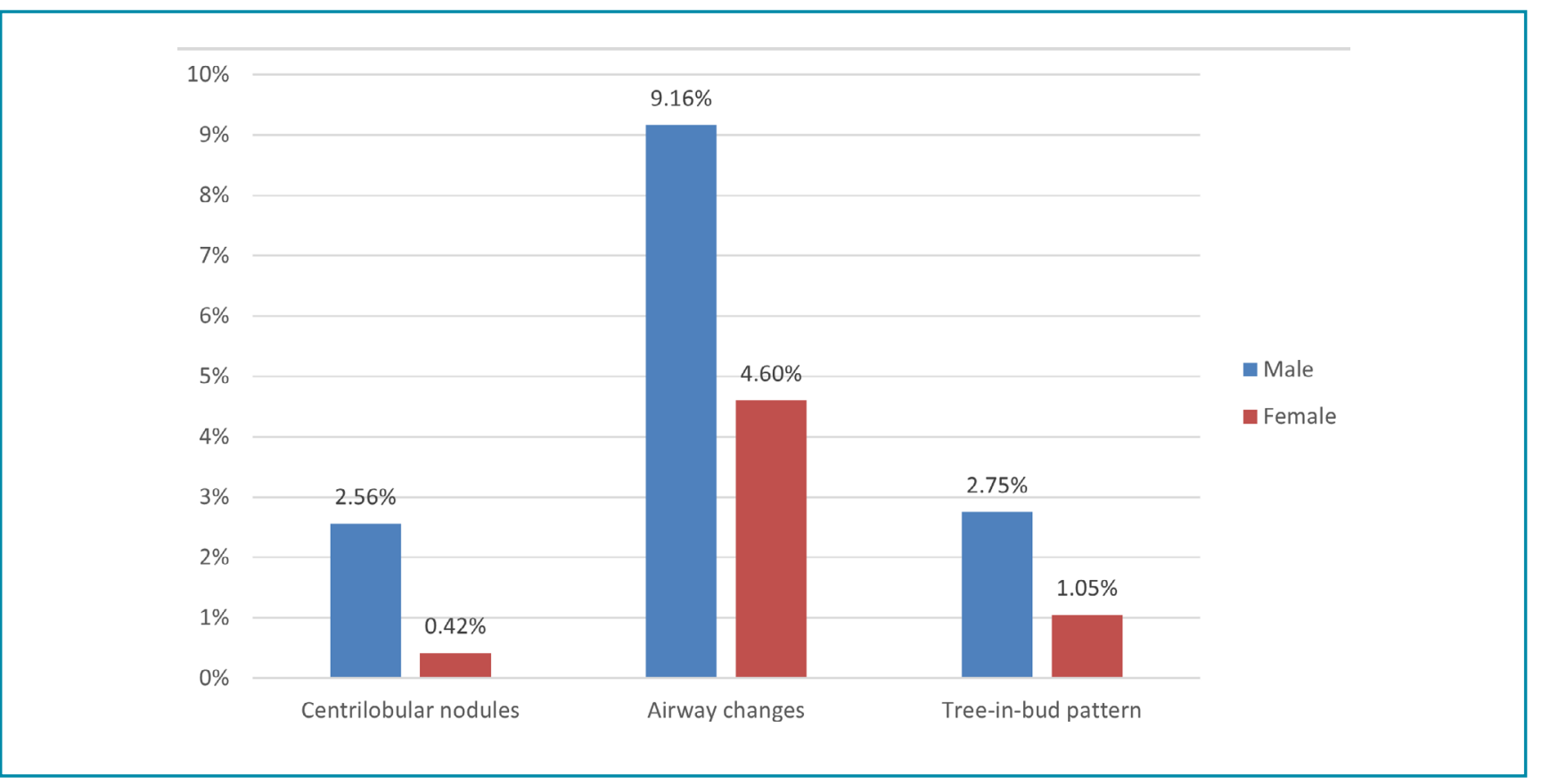

Figure 2. Statistically significant findings by gender.

Table 2. Distribution of parenchymal abnormalities according to age.

\begin{tabular}{|c|c|c|c|c|c|c|c|c|c|c|c|c|c|c|c|c|c|c|c|}
\hline & \multicolumn{2}{|c|}{$0-9$ age } & \multicolumn{2}{|c|}{$10-19$ age } & \multicolumn{2}{|c|}{ 20-29 age } & \multicolumn{2}{|c|}{$30-39$ age } & \multicolumn{2}{|c|}{ 40-49 age } & \multicolumn{2}{|c|}{ 50-59 age } & \multicolumn{2}{|c|}{$60-69$ age } & \multicolumn{2}{|c|}{$70-79$ age } & \multicolumn{2}{|c|}{$\geq 80$ age } & \multirow{2}{*}{$p$} \\
\hline & $\mathrm{n}$ & $\%$ & $n$ & $\%$ & $\mathrm{n}$ & $\%$ & $n$ & $\%$ & $n$ & $\%$ & $n$ & $\%$ & $n$ & $\%$ & $n$ & $\%$ & $\mathrm{n}$ & $\%$ & \\
\hline $\begin{array}{l}\text { Ground-glass } \\
\text { opacity }\end{array}$ & 4 & (66.67) & 10 & (27.78) & 54 & (36.24) & 131 & (60.65) & 161 & $(72.85)$ & 124 & $(83.78)$ & 103 & (93.64) & 85 & $(93.41)$ & 43 & (91.49) & $<0.001$ * \\
\hline Consolidation & 3 & $(50.00)$ & 4 & (11.11) & 24 & (16.11) & 54 & $(25.00)$ & 61 & $(27.60)$ & 62 & $(41.89)$ & 57 & $(51.82)$ & 55 & $(60.44)$ & 30 & (63.83) & $<0.001$ * \\
\hline $\begin{array}{l}\text { Intra-interlobular } \\
\text { septal thickening }\end{array}$ & & & & & 2 & $(1.34)$ & 16 & $(7.41)$ & 17 & $(7.69)$ & 26 & (17.57) & 33 & $(30.00)$ & 40 & (43.96) & 18 & (38.30) & $<0.001 *$ \\
\hline $\begin{array}{l}\text { Thin reticular } \\
\text { pattern }\end{array}$ & & & & & 2 & $(1.34)$ & 6 & $(2.78)$ & 15 & (6.79) & 11 & (7.43) & 25 & (22.73) & 21 & (23.08) & 6 & (12.77) & $<0.001 *$ \\
\hline $\begin{array}{l}\text { Centrilobular } \\
\text { nodules }\end{array}$ & & & & & 4 & $(2.68)$ & & & 3 & (1.36) & 2 & (1.35) & 3 & $(2.73)$ & 3 & (3.30) & 1 & $(2.13)$ & 0.408 \\
\hline $\begin{array}{l}\text { Intraparenchymal } \\
\text { vascular } \\
\text { enlargement }\end{array}$ & & & & & 1 & $(0.67)$ & 3 & (1.39) & 12 & $(5.43)$ & 3 & $(2.03)$ & 5 & $(4.55)$ & 2 & $(2.20)$ & 2 & $(4.26)$ & 0.105 \\
\hline Halo sign & & & 1 & $(2.78)$ & 5 & (3.36) & 8 & (3.70) & 2 & $(0.90)$ & 2 & (1.35) & 1 & $(0.91)$ & & & 1 & $(2.13)$ & 0.323 \\
\hline Reverse halo sign & & & & & 1 & $(0.67)$ & 2 & $(0.93)$ & 4 & $(1.81)$ & 7 & (4.73) & 1 & $(0.91)$ & 2 & $(2.20)$ & & & 0.142 \\
\hline Fibrosis & & & & & & & 7 & (3.24) & 6 & $(2.71)$ & 12 & (8.11) & 4 & (3.64) & 9 & $(9.89)$ & 10 & (21.28) & $<0.001 *$ \\
\hline Pleural effusion & & & & & & & 1 & $(0.46)$ & 2 & $(0.90)$ & 2 & $(1.35)$ & 3 & $(2.73)$ & 7 & (7.69) & 10 & (21.28) & $<0.001 *$ \\
\hline $\begin{array}{l}\text { Mediastinal } \\
\text { lymphadenopathy }\end{array}$ & & & & & 3 & (2.01) & 1 & $(0.46)$ & 2 & $(0.90)$ & 4 & $(2.70)$ & 11 & (10.00) & 7 & (7.69) & 7 & (14.89) & $<0.001$ * \\
\hline Air bubble sign & & & & & 2 & (1.34) & & & 2 & $(0.90)$ & 2 & (1.35) & 5 & (4.55) & 8 & (8.79) & 2 & $(4.26)$ & $<0.001$ * \\
\hline Air bronchogram & & & & & 5 & (3.36) & 5 & $(2.31)$ & 9 & $(4.07)$ & 6 & $(4.05)$ & 8 & $(7.27)$ & 17 & (18.68) & 12 & (25.53) & $<0.001 *$ \\
\hline Airway changes & & & & & 4 & (2.68) & 7 & (3.24) & 8 & (3.62) & 6 & (4.05) & 14 & (12.73) & 21 & (23.08) & 12 & (25.53) & $<0.001$ * \\
\hline Pericardial effusion & & & & & & & & & & & 1 & $(0.68)$ & 1 & $(0.91)$ & 6 & $(6.59)$ & 3 & $(6.38)$ & $<0.001 *$ \\
\hline Tree-in-bud & & & & & 7 & $(4.70)$ & 5 & $(2.31)$ & 3 & (1.36) & 1 & $(0.68)$ & 2 & $(1.82)$ & & & 2 & (4.26) & 0.168 \\
\hline $\begin{array}{l}\text { Crazy-paving } \\
\text { pattern }\end{array}$ & & & & & 1 & $(0.67)$ & 6 & $(2.78)$ & 14 & $(6.33)$ & 18 & $(12.16)$ & 23 & (20.91) & 20 & (21.98) & 14 & (29.79) & $<0.001^{*}$ \\
\hline
\end{tabular}

${ }^{*} p<0.05$ is statistically significant. 


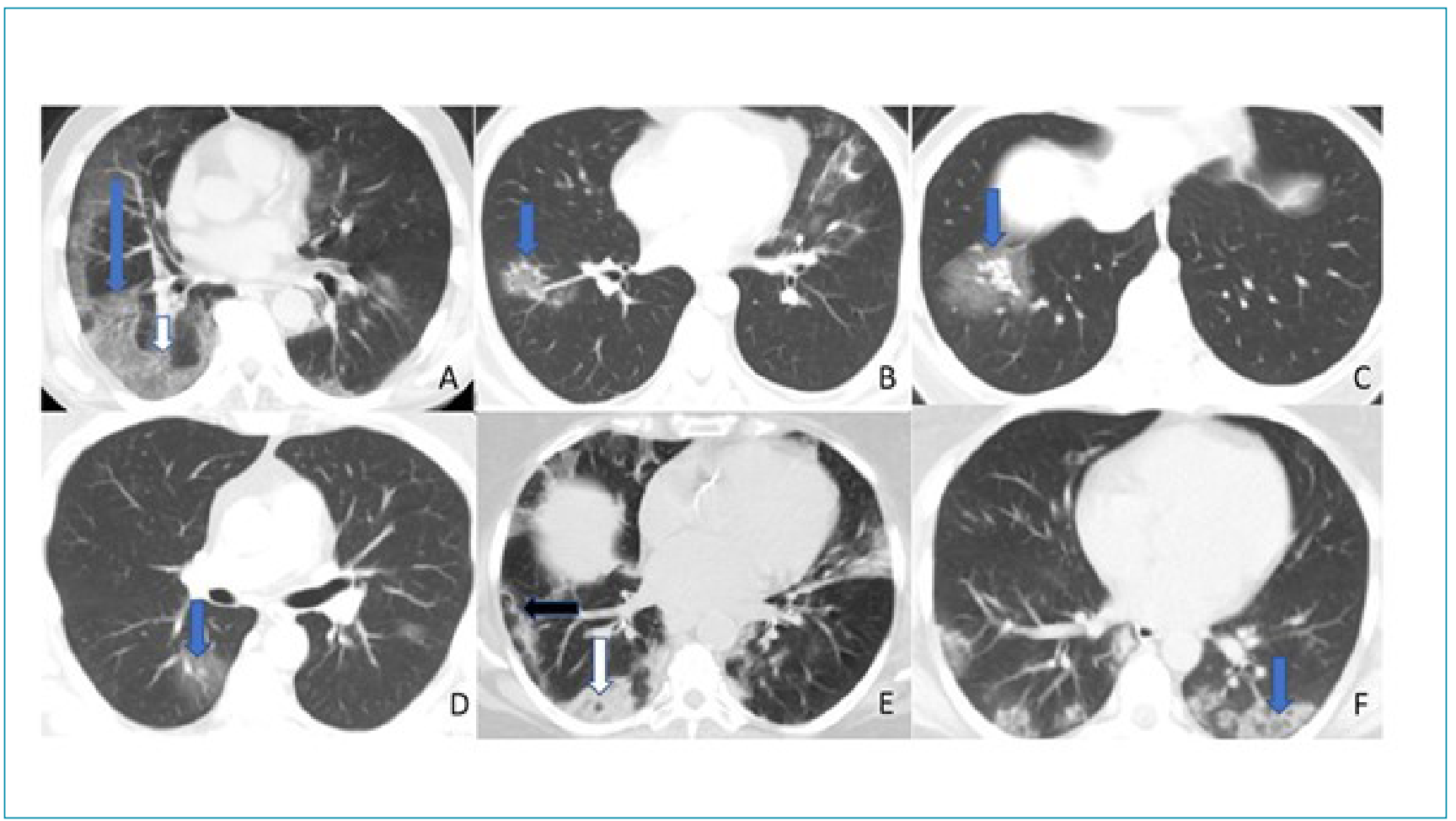

Figure 3. (A) Multiple ground-glass opacities and consolidation with a thickened intra-interlobular septum (blue arrow). The air bronchogram sign (white arrow). (B) Reversed halo sign in the right lower lobe upper segment (blue arrow). (C) Halo sign, central denser consolidation surrounded by ground-glass opacity of crescent shape in right lower lobe (blue arrow). (D) Multiple patchy ground-glass opacities with consolidation scattered in the peripheral zone of lower lobe, presenting with poorly defined boundaries and vascular thickening (blue arrow). (E) Ground-glass opacities with the air bubble sign in the upper lobe of the right lung (white arrow) and ground-glass opacities with subpleural line in the right inferior lobe (black arrow). (F) Crazy-paving pattern, multiple ground-glass opacities distributed in the peripheral area, ground-glass opacities with consolidation, and thickened intralobular septum (blue arrow).

\section{DISCUSSION}

This retrospective study showed that the CT manifestations of patients of different ages and gender were not exactly the same. Therefore, determining their CT features can help deepen our understanding of differences in disease characteristics between different age groups and can assist in clinical diagnosis and treatment decision-making.

In patients infected with COVID-19, the mortality increases with the increase in age, which is attributed to the higher incidence of comorbid diseases in advanced ages ${ }^{9}$. However, the disease can also have a severe course in young people without any comorbidity or may present with mild symptoms in elderly individuals with certain comorbidities. Zhu et al. found no significant difference in the development and progression stages of the disease in young and elderly patients ${ }^{10}$. However, many previous studies show that being old, male, and having comorbid diseases are poor prognostic factors for COVID- $19^{11}$. Li et al. stated that the mortality rate among elderly patients was significantly higher in males but they also noted no significant difference in mortality according to age ${ }^{12}$.

Chest $\mathrm{CT}$ is the key imaging modality in the early diagnosis of COVID-19, with the most common CT findings being patchy subsegmental or multiple GGOs with bilateral segmentation $^{13}$. In all ages, the most common finding of COVID-19 pneumonia is GGO. The multiple and bilateral incidence of GGO was found to be statistically significant in elderly patients. In addition, a strong relationship was observed between age and the number of involved lobes ${ }^{14}$. Wang et al. reported that non-subpleural distribution, single lesions, and limited number of involved lobes were common in patients under 40 years of age ${ }^{15}$. Similarly, the most common finding in this study was GGO, with its incidence significantly increasing over the age of 60 years.

Song et al. stated that consolidation was seen more frequently in young patients and GGO in elderly patients, and the area of the lungs involved was more in elderly patients ${ }^{16}$. 
However, we found that the consolidation incidence increased over the age of 70 years.

In this study, the halo sign was more common in 30-39 years. Xia et al. stated that the halo sign accounted for up to $50 \%$ of pediatric cases as a typical sign in pediatric patients ${ }^{17}$. In this study, the rate of the halo sign in pediatric patients was only $2.78 \%$. The incidence of subpleural line and pleural thickness is higher in elderly patients than young people ${ }^{10}$. Wang et al. reported that moderate pleural thickening and bronchiectasis were more common in elderly patients ${ }^{15}$. Gu et al. stated that the interlobular thickening and honeycomb pattern findings were more common in elderly people ${ }^{18}$. In this study, the thin reticular pattern finding was more common in the patients aged 60-79 years, and airway changes and the crazy-paving pattern were more common over 80 years. The higher rates of both pleural and airway findings in elderly patients were consistent with the literature.

Gu et al. reported that nodules were more common in younger people ${ }^{18}$. We found no significant difference in terms of nodule incidence. In this study, the more common finding in the young age group was the tree-in-bud pattern, which was mostly seen in the 20-29 years age group.

According to the reports of the World Health Organization, COVID-19 infection is more common in men, but the effect of gender difference on prognosis remains unclear ${ }^{19}$. Ueyama et al. stated that COVID-19 infection was more severe in men, but there was no significant difference in mortality between the genders ${ }^{20}$. Gu et al. found no significant difference between genders in their study with 50 patients ${ }^{18}$. In this study, the more common findings in men compared with women were centrilobular nodules, airway changes, and tree-in-bud pattern. The higher frequencies of all these three findings in men were statistically significant.
There are some limitations in this study. We used single imaging for each patient and we did not evaluate the progress of COVID-19 with dynamic imaging. This study mostly emphasized on the CT imaging features of COVID-19 and the differences between age groups and gender. We did not explore the relationship between the COVID-19 imaging findings and disease prognosis or mortality.

\section{CONCLUSION}

Multiple lobes being affected, subpleural lesions, crazy-paving pattern, bronchodilation, and pleural thickening are more common COVID-19 findings in elderly patients than in young people. Each age group has its own characteristics, and having knowledge on these features can help better understand the disease, make accurate diagnosis, and manage follow-up. There were significant differences in the imaging findings of the patients with confirmed COVID-19 pneumonia by age and gender. These differences became more evident in advanced ages. We consider that this study will contribute to the literature in terms of showing that the COVID-19 disease progresses asymptomatically or mildly in younger ages and progresses seriously in advanced ages, with the mortality rate being markedly higher among the elderly patients.

\section{AUTHORS' CONTRIBUTIONS}

AK: Data curation, Investigation, Resources, Validation, Writingoriginal draft. ZK: Formal analysis, Methodology, Software. OT: Project administration, Writing-review \& editing. YG: Visualization. MHO: Conceptiualization. OFA: Supervision. FG: Finding acquisition.

\section{REFERENCES}

1. Zhu N, Zhang D, Wang W, Li X, Yang B, Song J, et al. A novel coronavirus from patients with pneumonia in China, 2019. N Engl J Med. 2020;382(8):727-33. https://doi.org/10.1056/ NEJMoa2001017

2. Xu Z, Shi L, Wang Y, Zhang J, Huang L, Zhang C, et al. Pathological findings of COVID-19 associated with acute respiratory distress syndrome. Lancet Respir Med. 2020;8(4):420-2. https://doi. org/10.1016/S2213-2600(20)30076-X

3. Zhou F, Yu T, Du R, Fan G, Liu Y, Liu Z, et al. Clinical course and risk factors for mortality of adult inpatients with COVID-19 in Wuhan, China: a retrospective cohort study. Lancet. 2020;395(10229):1054-62. https://doi.org/10.1016/S01406736(20)30566-3
4. Jalaber $C$, Lapotre $T$, Morcet-Delattre $T$, Ribet $F$, Jouneau $S$, Lederlin M. Chest CT in COVID-19 pneumonia: a review of current knowledge. Diagn Interv Imaging. 2020;101(7-8):431-7. https://doi.org/10.1016/j.diii.2020.06.001

5. Xie X, Zhong Z, Zhao W, Zheng C, Wang F, Liu J. Chest CT for typical coronavirus disease 2019 (COVID-19) pneumonia: relationship to negative RT-PCR testing. Radiology. 2020;296(2):E41-E45. https://doi.org/10.1148/radiol.2020200343

6. Fang $Y$, Zhang $H$, Xie J, Lin $M$, Ying $L$, Pang $P$, Ji $W$. Sensitivity of chest CT for COVID-19: comparison to RT-PCR. Radiology. 2020;296(2):E115-E117. https://doi.org/10.1148/ radiol.2020200432

7. Awulachew E, Diriba K, Anja A, Getu E, Belayneh F. Computed tomography (CT) imaging features of patients with COVID- 
19: systematic review and meta-analysis. Radiol Res Pract. 2020;2020:1023506. https://doi.org/10.1155/2020/1023506

8. Bernheim A, Mei X, Huang M, Yang Y, Fayad ZA, Zhang $\mathrm{N}$, et al. Chest $C T$ findings in coronavirus disease-19 (COVID-19): relationship to duration of infection. Radiology. 2020;295(3):200463. https://doi.org/10.1148/radiol.2020200463

9. Han X, Cao Y, Jiang N, Chen Y, Alwalid O, Zhang X, et al. Novel coronavirus disease 2019 (COVID-19) pneumonia progression course in 17 discharged patients: comparison of clinical and thin-section computed tomography features during recovery. Clin Infec Dis. 2020;71(15):723-31. https://doi.org/10.1093/ cid/ciaa271

10. Zhu T, Wang $Y$, Zhou S, Zhang N, Xia L. A comparative study of chest computed tomography features in young and older adults with corona virus disease (COVID-19). J Thorac Imaging. 2020;35(4):W97-W101. https://doi.org/10.1097/ RTI.0000000000000513

11. Yang $X, Y u Y, X u$ J, Shu $H, X i a ~ J, ~ L i u ~ H$, et al. Clinical course and outcomes of critically ill patients with SARS-CoV-2 pneumonia in Wuhan, China: a single-centered, retrospective, observational study. Lancet Respir Med. 2020;8(5):475-81. https://doi.org/10.1016/\$2213-2600(20)30079-5

12. Li W, Fang Y, Liao J, Yu W, Yao L, Cui H, et al. Clinical and CT features of the COVID-19 infection: comparison among four different age groups. Eur Geriatr Med. 2020;11(5):843-50. https://doi.org/10.1007/s41999-020-00356-5

13. Jin $Y H$, Cai L, Cheng ZS, Cheng H, Deng T, Fan YP, et al. A rapid advice guideline for the diagnosis and treatment of 2019 novel coronavirus (2019-nCoV) infected pneumonia (standard version). Mil Med Res. 2020;7(1):4. https://doi.org/10.1186/ s40779-020-0233-6
14. Liu KC, Xu P, Lv WF, Qiu XH, Yao JL, Gu JF, et al. CT manifestations of coronavirus disease-2019: a retrospective analysis of 73 cases by disease severity. Eur J Radiol. 2020;126:108941. https://doi.org/10.1016/j.ejrad.2020.108941

15. Wang J, Zhu X, Xu Z, Yang G, Mao G, Jia Y, et al. Clinical and CT findings of COVID-19: differences among three age groups. BMC Infect Dis. 2020;20(1):434. https://doi.org/10.1186/ s12879-020-05154-9

16. Song F, Shi N, Shan F, Zhang Z, Shen J, Lu H, et al. Emerging 2019 novel coronavirus (2019-nCoV) pneumonia. Radiology. 2020;295(1):210-7. https://doi.org/10.1148/radiol.2020200274

17. Xia W, Shao J, Guo Y, Peng X, Li Z, Hu D. Clinical and CT features in pediatric patients with COVID-19 infection: different points from adults. Pediatr Pulmonol. 2020;55(5):1169-74. https://doi.org/10.1002/ppul.24718

18. Gu Q, Ouyang X, Xie A, Tan X, Liu J, Huang F, et al. A retrospective study of the initial chest $C T$ imaging findings in 50 COVID-19 patients stratified by gender and age. J Xray Sci Technol. 2020;28(5):875-84. https://doi.org/10.3233/ XST-200709

19. World Health Organization. COVID-19 situation update for the WHO European region: data for the week of 16-22 March 2020 (Epi week 12). 2020 [cited on 2020 Mar 27]. Available from: https://www.euro.who.int/_data/assets/pdf_file/0004/435316/ week12-COVID-19-surveillance-report.pdf.

20. Ueyama H, Kuno T, Takagi H, Krishnamoorthy P, Vengrenyuk $Y$, Sharma SK, et al. Gender difference is associated with severity of coronavirus disease 2019 infection: an insight from a meta-analysis. Crit Care Explor. 2020;2(6):e0148. https:// doi.org/10.1097/CCE.0000000000000148 